\title{
On the global Hadamard parametrix in QFT and the signed squared geodesic distance defined in domains larger than convex normal neighbourhoods
}

\author{
Valter Moretti ${ }^{1}$
}

Received: 14 July 2021 / Revised: 19 August 2021 / Accepted: 6 September 2021 /

Published online: 9 October 2021

(C) The Author(s) 2021

\begin{abstract}
We consider the global Hadamard condition and the notion of Hadamard parametrix whose use is pervasive in algebraic QFT in curved spacetime (see references in the main text). We point out the existence of a technical problem in the literature concerning well-definedness of the global Hadamard parametrix in normal neighbourhoods of Cauchy surfaces. We discuss in particular the definition of the (signed) geodesic distance $\sigma$ and related structures in an open neighbourhood of the diagonal of $M \times M$ larger than $U \times U$, for a normal convex neighbourhood $U$, where $(M, g)$ is a Riemannian or Lorentzian (smooth Hausdorff paracompact) manifold. We eventually propose a quite natural solution which slightly changes the original definition by Kay and Wald and relies upon some non-trivial consequences of the paracompactness property. The proposed re-formulation is in agreement with Radzikowski's microlocal version of the Hadamard condition.
\end{abstract}

Keywords Hadamard parametrix $\cdot$ Hadamard states $\cdot$ Geodesical distance $\cdot$ Normal convex neighborhood

Mathematics Subject Classification $81 \mathrm{~T} 20 \cdot 53 \mathrm{~B} 30 \cdot 53 \mathrm{C} 50 \cdot 83 \mathrm{C} 47 \cdot 53 \mathrm{~B} 21$

\section{Introduction}

The use of Hadamard states is nowadays pervasive in algebraic QFT (aQFT) in curved spacetime (see, e.g. [1,2,7-12,16,17,27,28,35] and [3] for a recent survey on aQFT). The rigorous definition of Hadamard state in terms of short-distance behaviour of the

Valter Moretti

valter.moretti@unitn.it

1 Department of Mathematics, University of Trento, and INFN-TIFPA, via Sommarive 14, I-38123

Povo (Trento), Italy 
two-point function was stated in the celebrated paper [19] by Kay and Wald for the first time. Some years later, that technically complex definition was translated into the language of microlocal analysis within a pair of nice papers by Radzikowski [32,33].

The original geometric definition of [19] of a global Hadamard parametrix has been exploited for instance to deal with rigorous interpretations of the Hawking radiation, see [29] and the recent interesting paper [22]. Just to mention some other applications of the Hadamard parametrix in aQFT (the following list of examples is by no means exhaustive), we can say that it plays a crucial role in the definition of locally covariant Wick powers [16,20], including the definition of the stress-energy tensor operator [27]. The Hadamard parametrix has been also employed in the study of quantum energy inequalities [10]. It has been also used in semiclassical approaches to the quantum gravity and cosmological applications [7,24].

Locally (and a bit roughly) speaking, in a globally hyperbolic four-dimensional spacetime $(M, g)$, an algebraic state $\omega$ of a real scalar Klein-Gordon quantum field is of Hadamard type if its two-point function $\Lambda_{\omega}$ has the Hadamard short-distance singularity,

$$
\Lambda_{\omega}(x, y)=\frac{1}{(2 \pi)^{2}}\left(\frac{\Delta(x, y)^{1 / 2}}{\sigma(x, y)}+v(x, y) \ln \sigma(x, y)\right)+H_{\omega}(x, y)
$$

when viewed as an integral kernel (see Sect. 3 for some technical details here disregarded). $H_{\omega}$ is a smooth function depending on the state $\omega$, whereas $\Delta, v, \sigma$ are universal geometric objects constructed out of the local geometry only. In particular, $\sigma(x, y)$ is the so-called signed squared geodesic distance of $x, y \in M$. It is defined as the squared length—with the appropriate sign-of the geodesic segment joining $x$ and $y$. The so-called Hadamard parametrix is the singular universal part $\Lambda_{\omega}(x, y)-H(x, y)$.

Since there are many geodesics, in principle, joining $x$ and $y$, a standard possibility is to assume that the identity above is true in a normal convex neighbourhood ${ }^{1}$ (see Sect. 2). This is an open set $U$ such that every pair of points $x, y \in U$ can be joined by a unique geodesic segment $\gamma:[0,1] \rightarrow M$ that belongs to the set: $\gamma([0,1]) \subset U$. This elementary precaution is not enough however in the global definition discussed in [19]. It is because (1) is assumed to be valid for pairs $(x, y)$ contained in many normal convex neighbourhoods. In principle, this gives rise to a cumbersome many-valued function $\sigma$. This is one of the most difficult technical issues tackled in [19].

Remark 1 If the spacetime points $x$ and $y$ belong to many convex normal neighbourhoods, different choices of such a neighbourhood may not only lead to different values for $\sigma(x, y)$ around $(x, y)$, but also to different singularity structures as determined by the Hadamard parametrix (1). Therefore, in principle, the introduced issue not only affects the construction of the Hadamard parametrix, but also the singularity structure that the Hadamard condition is assumed to describe. In particular, Hadamard states may not satisfy (1) in every convex normal neighbourhood. (See also the end of Example 2.)

\footnotetext{
1 A weaker requirement is that the identity is valid in a normal neighbourhood of one of the points.
} 
If $x$ and $y$ are causally related, a natural choice of $U$ for the given $x, y$ exists which solves the problem of the definition of $\sigma(x, y)$ and it was adopted in [19] (see Remark 13 for more details). $U$, if it exists, is a normal convex neighbourhood that simultaneously includes $x, y$, and their causal double cone $J(x, y)$ (see (7)). Obviously, every other normal convex neighbourhood $U^{\prime}$ which both includes $x, y$, and $J(x, y)$ must also contain the causal geodesic $\gamma$ joining $x$ and $y$ in $U$. As $U^{\prime}$ is convex, $\gamma$ is also its own geodesic $\gamma^{\prime}$ joining $x$ and $y$ in $U^{\prime}$. There is, in fact, only one geodesic segment (parametrized in $[0,1]$ ) joining a pair of causally related points $x$ and $y$ in common for the subfamily of the said normal convex sets. For these pairs $(x, y), \sigma(x, y)$ can be therefore unambiguously defined.

Physics is properly reflected by the family of causal geodesics, but a mathematically coherent definition of the Hadamard parametrix needs to consider also non-causal geodesics: the non-causal ones "arbitrarily close" to the causal ones. For technical reasons, in [19] $\sigma$ was therefore also required to be smooth and well-defined in a neighbourhood $\mathscr{O}$ of that special family of causally related pairs $(x, y)$. We stress that the neighbourhood $\mathscr{O}$ must also contain non-causally connected pairs. The argument used to give a non-ambiguous definition of $\sigma$ cannot be used for those pairs. The existence of $\mathscr{O}$ with a non-ambiguous extension of the definition of $\sigma$ was assumed in [19] and also in [32,33] without a proof. In this author's view, it remains a gap in the whole construction. This work is devoted to that gap.

Referring to Remark 1, one eventually sees that the problem of the definition of $\sigma$ for non-causally connected pairs actually affects the definition of the Hadamard parametrix in (1), but not its singularity structure (see Remark 20).

We shall not try to directly prove the existence of that $\mathscr{O}$. Our solution relies on a thin refinement of the definition of Hadamard parametrix which is possible thanks to a consequence of the paracompactness property. The final new definition of Hadamard state, which is a quite slight modification of the original definition in [19], though it is based on a non-trivial topological result, turns out to be in agreement with the microlocal version of the Hadamard condition.

To achieve our final goal, in the first part of the paper, we shall focus on the more abstract and mathematically minded problem of a well-posed definition of $\sigma$ (and related geometric objects) in a neighbourhood of the diagonal of $M \times M$. This issue is the core of the problem with the Hadamard parametrix, but it may have other applications in mathematical physics, so that it deserves a separate study.

Example 2 A concrete elementary illustration of the problems one faces when trying to define $\sigma\left(x, x^{\prime}\right)$ in a non-trivial spacetime is the following one ${ }^{2}$. Consider the spacetime $(M, g)$ constructed out of the $1+1$ Minkowski spacetime periodically identified under $(t, x) \mapsto(t, x+2 L)(c=1)$. Differently from the Minkowski space, $M$ is not normal convex in its own right. To define $\sigma\left(x, x^{\prime}\right)$, one is therefore forced to make a choice of a normal convex open set containing $x$ and $x^{\prime}$.

Let $x=(0, L / 2)$ and $x^{\prime}=(L / 2, L) \equiv(L / 2,-L)$. These points are causally related and $J\left(x, x^{\prime}\right)$ (a null line segment) can be thickened up to become a normal convex neighbourhood $U$. We wish to define the function $\sigma$ near $\left(x, x^{\prime}\right)$. Let us first

\footnotetext{
2 This illustration is a straightforward re-elaboration of an example proposed by Fewster to the author in a private communication.
} 
consider nearby points that are still causally connected. In particular, let $y=x$ and $y^{\prime}=$ $(L / 2, L-\epsilon)=(L / 2,-L-\epsilon)$ with $0<\epsilon \ll L$. These are causally related and near to $\left(x, x^{\prime}\right)$. In the considered case, we can also assume, enlarging $U$ if necessary, that $U \supset J\left(y, y^{\prime}\right)$. We can create further normal convex neighbourhoods which include $\left(y, y^{\prime}\right)$ by

(i) thickening the line segment (in $\left.\mathbb{R}^{2}\right)$ between $(0, L / 2)$ and $(L / 2, L-\epsilon)$ which is a timelike geodesic between $y$ and $y^{\prime}$. It produces a convex neighbourhood $U^{\prime}$ which we can assume to satisfy again $U^{\prime} \supset J\left(y, y^{\prime}\right)$. The line segment between $(0, L / 2)$ and $(L / 2, L-\epsilon)$ belongs to both $U$ and $U^{\prime}$. Consequently, $\sigma\left(y, y^{\prime}\right)$ referred to $U$ must coincide with $\sigma\left(y, y^{\prime}\right)$ referred to $U^{\prime}$.

(ii) thickening the line segment between $(0, L / 2)$ and $(L / 2,-L-\epsilon)$ which is a spacelike geodesic between $y$ and $y^{\prime}$. In this case, we obtain a value for $\sigma\left(y, y^{\prime}\right)$ different from the one computed in $U$.

This illustrates why, when defining $\sigma\left(y, y^{\prime}\right)$ in the causally connected case, the condition on the geodesically convex neighbourhood that it contains $J\left(y, y^{\prime}\right)$ permits to select a common notion of distance.

Next, consider the causally disconnected case. Let $y=x=(0, L / 2)$ and $y^{\prime}=$ $(L / 2, L+\epsilon)=(L / 2,-L+\epsilon)$ with $0<\epsilon \ll L$. These are not causally connected. We can still create normal convex neighbourhoods containing $y$ and $y^{\prime}$ by thickening the line segment between $(0, L / 2)$ and $(L / 2, L+\epsilon)$ or the one between $(L / 2,-L+\epsilon)$ and $(0, L / 2)$ giving spacelike geodesics of differing lengths and different values of $\sigma\left(y, y^{\prime}\right)$. There are actually infinitely many other possibilities that can be obtained using other image points $(L / 2,(2 n+1) L+\epsilon), n \in \mathbb{Z}$, throughout a very slim thickening of these segments, wrapping on the cylinder without any self-intersection.

An explicit illustration of the content of Remark 1 arises by referring to the example above and considering the timelike related points $p=(0,0)$ and $p_{0}=(2 L, 0)$, which can also be connected by null geodesics. Thickening a timelike or a null geodesic from $p$ to $p_{0}$ to a convex normal neighbourhood leads to distinct singularity structures.

\section{Extension of the signed squared geodesic distance and related structures}

Smooth manifolds are hereafter assumed to be Hausdorff and paracompact ${ }^{3}$. We adopt the Lorentzian signature $(-,+, \cdots,+)$ and we follow [31] concerning basic definitions, notation, and results in the theory of Lorentzian manifolds (see [26] for an up-to-date general review).

\footnotetext{
${ }^{3}$ For a topological space locally homeomorphic to $\mathbb{R}^{n}$ and equipped with a smooth atlas, (a) the existence of a smooth Lorentz metric implies paracompactness (this is standard in the Riemannian case and it remains true with a more elaborated proof in the indefinite case), but not Hausdorffness; (b) when the space is connected, 2nd countability and paracompactness are equivalent. Thus, the usual requirements for spacetimes (existence of a Lorentz metric and connectedness) imply that all the subtle required topological conditions hold (paracompactness, 2nd countability, Lindelöf) but Hausdorffness, which must be imposed explicitly.
} 


\subsection{Normal convex sets and the local definition of the (signed) squared geodesic distance $\sigma$}

Following Chapter 5 of [31], exp : D $\subset T M \rightarrow M$ will denote the standard exponential map associated to the geodesic flow of $g$ of a smooth Riemannian or Lorentzian manifold $(M, g)$. Its maximal domain $\mathcal{D}$ is a fibrewise starshaped open neighbourhood of the zero section of $T M$, and $\exp _{p}(v):=\exp (p, v)$ if $(p, v) \in \mathcal{D}$.

Definition 3 If $(M, g)$ is a Riemannian or Lorentzian smooth manifold, a normal convex neighbourhood $U$-also known as normal convex open set-is an open set $U \subset M$ such that, for every $q \in U$, there is a starshaped open neighbourhood $V_{q}^{(U)}$ of the origin of $T_{q} M$ such that $\exp _{q}: V_{q}^{(U)} \rightarrow U$ is a diffeomorphism.

Remark 4 (1) $U$ as above is geodesically starshaped with respect to every $p \in U$ : for every other $q \in U$ there is only one geodesic segment $\gamma_{p q}^{(U)}:[0,1] \rightarrow M$ such that both $\gamma_{p q}^{(U)}(0)=p, \gamma_{p q}^{(U)}(1)=q$ and $\gamma_{p q}^{(U)}([0,1]) \subset U$ are valid (Proposition 31 in chapter 3 of [31]). By definition of $\exp _{p}$, it holds $\gamma_{p q}^{(U)}(t)=\exp _{p}\left(t v_{q}\right)$ where $v_{q}:=\exp _{p}^{-1}(q)$ and $t \in[0,1]$.

(2) As $U$ is geodesically starshaped with respect to every point $p \in U$, it is not difficult to prove that $V_{p}^{(U)}$ in Def. 3 is completely determined by $U$ and $p \in U$.

(3) The set $\cup_{p \in U}\{p\} \times V_{p}^{(U)} \subset \mathcal{D}$ is open in $T M$. This is because the differential of the bijective map $U \times U \ni(p, q) \mapsto\left(p,\left(\left.\exp _{p}\right|_{V_{p}^{(U)}}\right)^{-1}(q)\right) \in \cup_{p \in U}\{p\} \times V_{p}^{(U)}$ is everywhere non-singular so that the map is open. exp : $\cup_{p \in U}\{p\} \times V_{p}^{(U)} \rightarrow U \times U$ is the inverse diffeomorphism and thus it is smooth (see in particular Lemma 9, Chap 5 [31] and comments around it).

A crucial result by Whitehead ${ }^{4}$ proves that (Proposition 7 Chapter 5 of [31] and its proof.)

Theorem 5 For a Riemannian or Lorentzian smooth manifold $(M, g)$, the family of normal convex open sets is not empty and forms a topological basis of $M$.

Among other important constructions, the Whitehead theorem and the properties of exp allow one to define the so-called (signed) squared geodesic distance also known as Synge's world function. If $U$ is an open normal convex set in $(M, g)$,

$$
\begin{aligned}
\sigma_{U}(p, q) & :=g_{p}\left(\dot{\gamma}_{p q}^{(U)}(0), \dot{\gamma}_{p q}^{(U)}(0)\right) \\
& = \pm\left(\int_{0}^{1} \sqrt{\left|g\left(\dot{\gamma}_{p q}^{(U)}(t), \dot{\gamma}_{p q}^{(U)}(t)\right)\right|} \mathrm{d} t\right)^{2} \text { for } \quad p, q \in U
\end{aligned}
$$

\footnotetext{
4 The definition of normal convex neighbourhoods and Whitehead's result are more generally true for smooth manifolds equipped with smooth affine connections [21], however in this paper we stick to the smooth Levi-Civita connection generated by $g$.
} 
where the sign - appears only if $g$ is Lorentzian and $\gamma_{p q}^{(U)}$ is timelike. This function is smooth in $U \times U$ because $\gamma_{p q}^{(U)}(t)=\exp _{p}\left(t\left(\left.\exp _{p}\right|_{V_{p}^{(U)}}\right)^{-1}(q)\right)$ is smooth in $[0,1] \times U \times U$, it being the restriction to $[0,1] \times U \times U$ of the composition of three smooth functions (defined on open sets): $\exp : \mathcal{D} \rightarrow M$, the multiplication with $t$, and a component of $U \times U \ni(p, q) \mapsto\left(p,\left(\left.\exp _{p}\right|_{V_{p}^{(U)}}\right)^{-1}(q)\right) \in \cup_{p \in U}\{p\} \times V_{p}$ according to (3) in Remark 4.

It is evident that $\sigma_{U}(p, q)$ strictly depends on the choice of the normal convex neighbourhood containing the points $p, q$. If there were another normal convex neighbourhood $U^{\prime} \ni p, q$, in general $\sigma_{U}(p, q) \neq \sigma_{U^{\prime}}(p, q)$ because the two sides refer to generally different geodesic segments: one stays in $U$ and the other stays in $U^{\prime}$, though both geodesics join $p$ and $q$. This fact prevents one from defining $\sigma$ as a global smooth function over $M \times M$.

\subsection{Assignment of geodesics around the diagonal of $M \times M$ and extension of $\sigma$ thereon}

A natural issue which pops out at this juncture is whether or not $\sigma$ can be more globally defined, at least in an open neighbourhood $\mathscr{A}$ of the diagonal $\Delta_{M}:=\{(p, p) \mid p \in M\}$ of $M \times M$.

The root of the problem is that, generally speaking, there are many geodesics connecting a pair of points $p, q$ and $\sigma(p, q)$ depends on the choice of one of those curves. One restricts to work in a "small" neighbourhood $\mathscr{A}$ of the diagonal of $M \times M$ because it seems that the choice should be easier if $p$ and $q$ are close to each other. (There are however results concerning really global definitions of $\sigma$, on the whole $M \times M$, when assuming suitable hypotheses on the topology of $M$ [5].) To address the issue above, one may therefore wonder if it is possible to define a jointly smooth assignment of geodesic segments $\gamma_{p q}(t)=\Gamma(t, p, q)$ where $t \in[0,1],(p, q)$ varies in a neighbourhood $\mathscr{A}$ of $\Delta_{M}$ and $\gamma_{p q}(0)=p, \gamma_{p q}(t)=q$. Indeed, equipped with such an assignment, $\sigma$ can be defined on $\mathscr{A}$ by direct use of (2).

Remark 6 If $(M, g)$ is Riemannian and its injectivity radius is positive, then other known ways exist to define a (smooth) notion of squared geodesic distance in a neighbourhood of the diagonal of $M \times M$ (see, e.g. [36] for the case of a bounded geometry manifold in particular). However, we refer here to the general case where $(M, g)$ may be Lorentzian, or Riemannian with zero injectivity radius.

An idea to construct $\Gamma$ and $\sigma$ in $\mathscr{A}$ (see also the discussion on p. 131 of [31]) relies on the insight that sufficiently small normal convex neighbourhoods are expected to have intersections which are normal convex as well. In that case, if $U \cap U^{\prime}$ is convex and both $p, q \in U, p, q \in U^{\prime}$, then the unique geodesic segment $\Gamma_{U}(t, p, q):=\gamma_{p, q}^{(U)}(t) \in U$, $t \in[0,1]$, joining them in $U$ coincides with the analogue $\Gamma_{U^{\prime}}(t, p, q):=\gamma_{p, q}^{U^{\prime}}(t) \in U^{\prime}$ joining $p$ and $q$ in $U^{\prime}$, since it is the unique geodesic segment joining $p$ and $q$ in $U \cap U^{\prime}$. Therefore $\Gamma_{U}(t, p, q)=\Gamma_{U \cap U^{\prime}}(t, p, q)=\Gamma_{U^{\prime}}(t, p, q)$. If a covering $\mathcal{C}$ of $M$ exists made of normal convex open sets such that $U, U^{\prime} \in \mathcal{C}$ implies that $U \cap U^{\prime}$ is convex as well, then a jointly smooth assignment of geodesic segments $\Gamma:[0,1] \times \mathscr{A} \rightarrow M$ 
joining the arguments $(p, q) \in \mathscr{A}$ (i.e. $\Gamma(0, p, q)=p$ and $\Gamma(1, p, q)=q)$ is welldefined and smooth on the open neighbourhood $\mathscr{A}$ of $\Delta_{M}$. It suffices to define

$$
\begin{aligned}
& \mathscr{A}:=\bigcup_{U \in \mathcal{C}} U \times U \\
& \text { if }(x, y) \in \mathscr{A}, \quad \Gamma(t, x, y):=\Gamma_{U}(t, x, y) \text { where } U \in \mathcal{C} \text { is such that } x, y \in U .
\end{aligned}
$$

Indeed, if $(x, y) \in \mathscr{A}$, then there must exist $U \in \mathcal{C}$ such that $x, y \in U$. Next, the right-hand side of (2) is well defined, since it does not depend on $U$ if there are other elements in $\mathcal{C}$ containing $x, y$ as pointed out above. $\Gamma$ is also jointly smooth on $\mathscr{A}$ because it is locally jointly smooth. In this way, an associated signed squared geodesic distance $\sigma: \mathscr{A} \rightarrow \mathbb{R}$ results to be well-defined and smooth because it is a composition of smooth functions:

$$
\sigma(p, q):=g_{p}\left(\left.\frac{\partial \Gamma}{\partial t}\right|_{(0, p, q)},\left.\frac{\partial \Gamma}{\partial t}\right|_{(0, p, q)}\right), \quad(p, q) \in \mathscr{A} .
$$

Definition 7 If $(M, g)$ is a smooth Riemannian or Lorentzian manifold, a strongly convex covering of $M$ is a covering $\mathcal{C}$ of $M$ made of normal convex open sets such that $C \cap C^{\prime}$ is normal convex if $C, C^{\prime} \in \mathcal{C}$ and $C \cap C^{\prime} \neq \varnothing$.

Existence of a strongly convex covering $\mathcal{C}$ is guaranteed when explicitly assuming Hausdorff and paracompactness hypotheses on $M^{5}$. In fact, paracompactness possesses an important technical feature discovered by A.H. Stone [37] (see also [25]).

Theorem 8 A topological space $X$ is Hausdorff and paracompact if and only if it is $T_{1}$ and every covering $\mathcal{C}$ of $X$ made of open sets admits $a^{*}$-refinement of open sets. That is another covering $\mathcal{C}^{*}$ of open sets such that, for every $V \in \mathcal{C}^{*}$,

$$
\bigcup\left\{V^{\prime} \in \mathcal{C}^{*} \mid V^{\prime} \cap V \neq \varnothing\right\} \subset U_{V} \text { for some } U_{V} \in \mathcal{C} \text {. }
$$

(Notice that $V \subset U_{V} \in \mathcal{C}$ in particular, so that a ${ }^{*}$-refinement is a refinement as well). This theorem implies the existence of the desired well-behaved covering $\mathcal{C}$ of normal convex open sets of $(M, g)$ (see also Lemma 10 in Chapter 5 of [31]).

Proposition 9 Let $(M, g)$ be a smooth (Hausdorff paracompact) Riemannian or Lorentzian manifold and $\mathcal{A}$ a covering of $M$ made of open sets (possibly $\mathcal{A}:=\{M\}$ ). Then, there exists a covering $\mathcal{C}$ of $M$ sets such that,

(a) $\mathcal{C}$ is a refinement of $\mathcal{A}$ (i.e. if $C \in \mathcal{C}$, then $C \subset U_{C} \in \mathcal{A}$ ) made of normal convex open sets;

\footnotetext{
5 This idea is sketched in Lemma 10 of Chapter 5 of [31] unfortunately with very few details and without explicitly referring to the crucial topological result of Theorem 8 .
} 
(b) $\mathcal{C}$ is a strongly convex covering of $M$.

Proof Using Theorem 5, consider the covering $\mathcal{C}_{0}$ made of all normal convex neighbourhoods that are subsets of the elements of $\mathcal{A}$. Exploiting Theorem 8, consider a refinement $\mathcal{C}_{0}^{*}$ of $\mathcal{C}_{0}$ satisfying, for every $V \in \mathcal{C}_{0}^{*}$,

$$
\bigcup\left\{V^{\prime} \in \mathcal{C}_{0}^{*} \mid V^{\prime} \cap V \neq \varnothing\right\} \subset C_{V} \text { for some } C_{V} \in \mathcal{C}_{0} .
$$

The proof concludes by defining $\mathcal{C}$ as the family of normal convex neighbourhoods contained within elements of $\mathcal{C}_{0}^{*}$ so that (a) is in particular true by construction. To prove (b), we start by observing that, if $C, C^{\prime} \in \mathcal{C}$, then $C \subset V$ and $C^{\prime} \subset V^{\prime}$ for some $V, V^{\prime} \in \mathcal{C}_{0}^{*}$; if furthermore $C \cap C^{\prime} \neq \varnothing$, we conclude that $V \cap V^{\prime} \neq \varnothing$ and thus $C \cup C^{\prime} \subset V \cup V^{\prime} \subset C_{V}$. Property (b) now comes easily using convex normality of $C_{V}$. First the intersection $C \cap C^{\prime}$ is open. Next, if $p, q \in C \cap C^{\prime}$, then the unique geodesic segment $\gamma:[0,1] \rightarrow C_{V}$ joining $p$ and $q$ is also completely included in $C \cap C^{\prime}$ since it must simultaneously stay in $C$ and $C^{\prime}$, they being normal convex as well. As a consequence, if $p \in C \cap C^{\prime}$, it necessarily holds $C \cap C^{\prime}=\exp _{p}\left(V_{p}^{\left(C \cap C^{\prime}\right)}\right)$ for some star-shaped open neighbourhood $V_{p}^{\left(C \cap C^{\prime}\right)}:=\left(\left.\exp _{p}\right|_{V_{p}^{(C)}}\right)^{-1}\left(C \cap C^{\prime}\right)$ of the origin of $T_{p} M$. Notice that $\left.\exp _{p}\right|_{V_{p}^{\left(C \cap C^{\prime}\right)}}: V_{p}^{\left(C \cap C^{\prime}\right)} \rightarrow C \cap C^{\prime}$ is a diffeomorphism because it is the restriction of the diffeomorphism $\left.\exp _{p}\right|_{V_{p}^{(C)}}: V_{p}^{(C)} \rightarrow C$. In summary, $C \cap C^{\prime}$ fulfils Definition 3 and the proof is over.

Collecting all results, we are in a position to state the main theorem of this section, concerning the existence of strongly convex coverings in particular, which also includes a (local) uniqueness statement.

Theorem 10 Let $(M, g)$ be a smooth (Hausdorff paracompact) Riemannian or Lorentzian manifold, and $\mathcal{C}$ a strongly convex covering of $M$. Then, the following facts hold.

(a) Defining the open neighbourhood $\mathscr{A} \supset \Delta_{M}$ as in (3) with respect to $\mathcal{C}$, the assignment of geodesic segments (2)

$\Gamma:[0,1] \times \mathscr{A} \ni(t, p, q) \rightarrow \gamma_{p, q}(t) \in M$ where $\gamma_{p, q}(0)=p$ and $\gamma_{p, q}(1)=q$, and the (signed) squared geodesic distance (5)

$$
\sigma(p, q):=g_{p}\left(\dot{\gamma}_{p, q}(0), \dot{\gamma}_{p, q}(0)\right) \text { for }(p, q) \in \mathscr{A}
$$

are well-defined and smooth on $\mathscr{A}$.

(b) If $\mathscr{A}^{\prime} \supset \Delta_{M}, \Gamma^{\prime}:[0,1] \times \mathscr{A}^{\prime} \rightarrow M$, and $\sigma^{\prime}: \mathscr{A}^{\prime} \rightarrow \mathbb{R}$ is another triple as in (a) but constructed out of another strongly convex covering $\mathcal{C}^{\prime}$, then there is an open set $\mathscr{A}^{\prime \prime} \subset M \times M$ such that

$$
\mathscr{A} \cap \mathscr{A}^{\prime} \supset \mathscr{A}^{\prime \prime} \supset \Delta_{M},\left.\quad \Gamma\right|_{[0,1] \times \mathscr{A}^{\prime \prime}}=\left.\Gamma^{\prime}\right|_{[0,1] \times \mathscr{A} \prime \prime},\left.\quad \sigma\right|_{\mathscr{A}}{ }^{\prime \prime}=\left.\sigma^{\prime}\right|_{\mathscr{A}}
$$


Proof (a) If $\mathscr{A}$ is as in (3), $\Gamma:[0,1] \times \mathscr{A} \rightarrow M$ defined as in (2) and $\sigma: \mathscr{A} \rightarrow \mathbb{R}$ defined as in (5) are well-defined and smooth as discussed in the paragraph before Eq. (3) and after Eq. (2). (b) Define a new covering $\mathcal{C}_{1}$ (a simultaneous refinement of $\mathcal{C}$ and $\mathcal{C}^{\prime}$ ) made of the sets $C \cap C^{\prime}$, for all choices of $C \in \mathcal{C}$ and $C^{\prime} \in \mathcal{C}^{\prime}$. According to Proposition 9 , define $\mathcal{C}^{\prime \prime}$ as a refinement of $\mathcal{C}_{1}$ made of normal convex neighbourhoods such that $U, U^{\prime} \in \mathcal{C}^{\prime \prime}$ implies that $U \cap U^{\prime}$ is empty or normal convex and define $\mathscr{A}^{\prime \prime}:=\cup_{U^{\prime \prime} \in \mathcal{C}^{\prime \prime}} U^{\prime \prime} \times U^{\prime \prime}$. By construction, both $\mathscr{A}^{\prime \prime} \subset \mathscr{A}$ and $\mathscr{A}^{\prime \prime} \subset \mathscr{A}^{\prime}$. Moreover, if $x, y \in \mathscr{A}^{\prime \prime}$ then we have both $x, y \in U^{\prime \prime} \subset U \in \mathcal{C}$ and $x, y \in U^{\prime \prime} \subset U^{\prime} \in \mathcal{C}^{\prime}$, so that $\Gamma(t, x, y)=\Gamma_{U}(t, x, y)=\Gamma_{U^{\prime \prime}}(t, x, y)=\Gamma_{U^{\prime}}(t, x, y)=\Gamma^{\prime}(t, x, y)$. The same fact holds for $\sigma$ and $\sigma^{\prime}$ in view of their definition (5) in terms of $\Gamma$ and $\Gamma^{\prime}$.

Definition 11 A triple $(\mathscr{A}, \Gamma, \sigma)$ as in in (a) of Theorem 10 is said to be subordinated to $\mathcal{C}$.

Remark 12 Strongly convex coverings are not an ad hoc artefact for the proposal of this work, but a natural and commonly used technical tool in Semi-Riemannian Geometry. The existence of this sort of geodesically convex coverings is a straightforward fact in Riemannian Geometry (see the elementary version of the sketch of proof above when $h=g$ ). The extension to Lorentzian manifolds is however not straightforward. In addition to the topological approach of Proposition 9, a purely geometric (in this sense perhaps more natural) proof of existence of strongly convex coverings for a Lorentzian geometry can be obtained along the following construction ${ }^{6}$. (What follows is however valid, with the same proof, when referring to the geodesic flow of a smooth affine connection $\Gamma$ on $M$ which is not the Levi-Civita connection of some metric.)

Let $(M, g)$ be a smooth connected (Hausdorff 2nd countable) Lorentzian manifold.

(i) Let $h$ be an auxiliary Riemannian metric on $M$ (which exists as a consequence of elementary results in Riemannian geometry [21]). $h$ can be chosen in order that the Riemannian manifold $(M, h)$ is complete [30] so that the $h$-injectivity radius at a given point $p \in M$ is a continuous function of $p$ (see, e.g. Prop. 10.37 in [23]). Consider the atlas of Riemannian normal coordinates $\left(U_{p}, \psi_{p}\right)$ centred on every $p \in M$ and referred to the Riemannian metric $h$.

(ii) Following the classic proof of the Whitehead theorem on the existence of the topological basis of convex normal neighbourhoods of $g$ [21], one sees that every $\psi_{p}$-coordinate ball $B_{r}^{h}(p)$ with centre $p$ is $g$-normal convex if the radius $r_{p}$ is sufficiently small. This would happen if referring to any atlas on $M$, but in the considered case the balls $B_{r}^{h}(p)$ are also geodesical balls with respect to $h$ and they are normal and convex with respect to $h$ for sufficiently small $r_{p}$. As is known, these balls are also metric balls of the natural metric space on $M$ induced by $h$ (the distance $d(p, q)$ is the inf of the $h$-length of the smooth curves joining $p$ and $q)$.

(iii) Then, one can choose a continuous function $\mu: M \rightarrow(0,+\infty)-$ with $2 \mu$ smaller than the $h$-radius of injectivity at each point $p \in M-$ such that the ball $B_{r}^{h}(p)$ with $0<r \leq 2 \mu(p)$ is $g$-normal convex for all $p \in M$.

\footnotetext{
6 The author is grateful to an anonymous referee for the comments contained in this remark, and to M. Sànchez for the idea of the alternative existence proof of strongly convex coverings sketched here.
} 
A strong $g$-convex covering is made of the family of all the balls $B_{\mu(p)}^{h}(p), p \in M$. Indeed, from elementary properties of balls in metric spaces, the intersection (assumed to be non-empty) of a pair of such balls with centres $p_{1}$ and $p_{2}$ is included in the ball of the centre $p_{1}$ and (bigger) radius $2 \mu(p)$. This ball is $g$-convex by construction.

\section{An issue with the global Hadamard condition and Hadamard parametrix}

Before addressing another issue still related to the well-definedness of $\sigma$ and associated structures, we summarize the relevant notions introduced by the milestone paper [19] where, for the first time, a rigorous definition of a Hadamard state was proposed and used by Kay and Wald. The definition was used in [19] (relying on previous work as [14] and [18]) to establish some important uniqueness results of QFT on a spacetime equipped with a bifurcate Killing horizon related to the KMS states of a real Klein-Gordon scalar field with the Hawking temperature. However, the definition of Hadamard state discussed therein applies to every (four-dimensional) globally hyperbolic spacetime. The notion of Hadamard states in Kay-Wald's approach relies upon the notion of Hadamard parametrix. The Hadamard condition on states can be nowadays formulated without a Hadamard parametrix using microlocal techniques as we shall briefly discuss later. It is however worth stressing that the notion of Hadamard parametrix remains a crucial technical tool for the construction of other important mathematical objects in QFT as the Wick powers in the locally covariant formulation (see in particular [16,20]).

\subsection{Hadamard states according to [19]}

If $(M, g)$ is a time-oriented smooth spacetime and $x, y \in M$,

$$
J(x, y):=\left(J^{-}(x) \cap J^{+}(y)\right) \cup\left(J^{-}(y) \cap J^{+}(x)\right),
$$

where $J^{ \pm}(S)$ are defined as in [31]. We say that $x, y$ are causally related in $(M, g)$ if $J(x, y) \neq \varnothing$. We henceforth assume that $(M, g)$ is four dimensional and globally hyperbolic.

Remark 13 If $x, y \in M$ are causally related in a globally hyperbolic spacetime $(M, g)$, then there is a causal geodesic segment joining them in view of Proposition 19 in Chapter 14 of [31]. This fact has a crucial consequence. If $x, y$ are causally related and both $U \supset J(x, y), U^{\prime} \supset J(x, y)$ for convex normal neighbourhoods $U, U^{\prime}$, then $\sigma_{U}(x, y)=\sigma_{U^{\prime}}(x, y)$. Indeed, the unique geodesic segments parametrized on $[0,1]$ connecting $x$ and $y$, respectively, in $U$ and $U^{\prime}$ must belong to $J(x, y) \subset U \cap U^{\prime}$ and thus they must coincide. This fact is throughout exploited in [19] and provides a well-defined notion of signed squared geodesic distance $\sigma(x, y)$ on the subset of 
$M \times M$

$$
\mathscr{Z}_{M}:=\{(x, y) \in M \times M \mid
$$

$x, y$ causally related $, J(x, y) \subset U, U$ normal convex neighbourhood $\}$.

The definition of Hadamard state according to [19] passes through the following four steps.

(H1) The so-called (global) Hadamard parametrix is defined in [19], for every natural $n$ and $\epsilon>0$, as

$$
\begin{aligned}
& G_{\epsilon}^{T, n}(x, y):=\frac{1}{(2 \pi)^{2}} \\
& \quad\left[\frac{\Delta(x, y)^{1 / 2}}{\sigma(x, y)+2 i \epsilon t(x, y)+\epsilon^{2}}+v_{n}(x, y) \ln \left(\sigma(x, y)+2 i \epsilon t(x, y)+\epsilon^{2}\right)\right], \\
& \quad(x, y) \in \mathscr{O} .
\end{aligned}
$$

Above $\mathscr{O} \supset \mathscr{Z}_{M}$ is an open set supposed to exist where $\sigma$ and $G_{\epsilon}^{T, n}$ are well defined, $t(x, y):=T(x)-T(y)$, where the smooth function $T: M \rightarrow \mathbb{R}$ is a temporal function ${ }^{7}$ increasing towards the future, the branch cut of the logarithm is taken along the negative real axis, and the function $\Delta(x, y)$ and $v_{n}(x, y)$ are known and defined in terms of $\sigma(x, y)$ and known recursion integrals along the geodesic segment $\gamma_{x y}$ connecting $x$ and $y$ (see, e.g. Appendix A of [27] and [15]).

Remark 14 If $\sigma(x, y)$ and the geodesic segment $\gamma_{x y}$ connecting $x$ and $y$ are well defined in some neighbourhood, then $\Delta(x, y)$ and $v_{n}(x, y)$ are completely determined in that neighbourhood. This happens in particular for $x, y \in U$ with $U$ normal convex neighbourhood.

(H2) Following [19], given a globally hyperbolic spacetime $(M, g)$ with a time orientation and a smooth spacelike Cauchy surface $\Sigma$, a normal neighbourhood $N$ of $\Sigma$ is an open set including $\Sigma$ and such that

(a) $\left(N,\left.g\right|_{N}\right)$ is a globally hyperbolic spacetime and $\Sigma$ is a Cauchy surface of it;

(b) $(x, y) \in N \times N$ are causally related in $(M, g)$ iff $(x, y) \in \mathscr{Z}_{M}$.

Lemma 2.2 of [19] proves the existence of a normal neighbourhood of any given Cauchy surface $\Sigma$.

(H3) Consider an open set $\mathscr{O}^{\prime} \subset N \times N$ which includes $\mathscr{Z}_{M} \cap(N \times N)$ (i.e. the set of causally related pairs $(x, y) \in N \times N)$ and such that its closure in $N \times N$ satisfies ${\overline{\mathscr{O}^{\prime}}}^{N \times N} \subset \mathscr{O}$. Finally, $\chi: N \times N \rightarrow \mathbb{R}$ is a smooth function such that $\chi(x, y)=1$ for $(x, y) \in{\overline{\mathscr{O}^{\prime}}}^{N \times N}$ and $\chi(x, y)=0$ for $(x, y) \notin \mathscr{O} \cap(N \times N)$.

(H4) With $\mathscr{O}, N, T, \chi$ as above, we can state the definition of Hadamard state.

\footnotetext{
7 That is, $d T=0$ is everywhere past-directed.
} 
Definition 15 An algebraic state $\omega$ on the (Weyl $C^{*}$ or ${ }^{*}$ ) algebra of a real scalar Klein-Gordon field on $(M, g)$ is said to be globally Hadamard according to [19] if the associated two-point function, i.e. a certain bilinear map [19] $\Lambda_{\omega}: C_{0}^{\infty}(M) \times$ $C_{0}^{\infty}(M) \rightarrow \mathbb{C}$, satisfies the following requirement

$$
\begin{aligned}
& \Lambda_{\omega}\left(F_{1}, F_{2}\right)=\lim _{\epsilon \rightarrow 0^{+}} \int_{N \times N} \Lambda_{\epsilon}^{T, n}(x, y) F_{1}(x) F_{2}(y) d \mu_{g}(x) d \mu_{g}(y), \\
& \forall F_{1}, F_{2} \in C_{0}^{\infty}(N),
\end{aligned}
$$

where $\mu_{g}$ is the natural measure induced by $g$ on $M$ and

$$
\Lambda_{\epsilon}^{T, n}(x, y)=\chi(x, y) G_{\epsilon}^{T, n}(x, y)+H^{n}(x, y)
$$

for every natural $n$ and some associated functions $H^{n} \in C^{n}(N \times N)$.

Remark 16 In [19], it is proved that Definition 15 is independent of $\mathscr{O}, N, \chi, \Sigma$. Yet, that independence proof assumes at various steps that $G_{\epsilon}^{T, n}(x, y)$ is well defined, not only on $\mathscr{Z}_{M}$, but also on $\mathscr{O}$ (and $\mathscr{O}^{\prime}$ ). In particular, $\sigma(x, y)$ is expected to have the standard behaviour in $\mathscr{O}: \sigma(x, y)>0$ if $x \neq y$ are not causally related. More precisely, $\sigma(x, y)$ is supposed to take the standard form $\sigma\left(x, x^{\prime}\right)=-\left(y^{0}\left(x^{\prime}\right)\right)^{2}+\sum_{\alpha=1}^{3}\left(y^{\alpha}\left(x^{\prime}\right)\right)^{2}$ in Riemannian normal coordinates $y^{0}, y^{1}, y^{2}, y^{3}$ centred at one of the entries (here $x$ ) also for non-causally related arguments.

Definition 15 was later proved to be equivalent to a certain microlocal version by a famous paper by M. Radzikowski [32], when assuming the requirement $\Lambda_{\omega} \in \mathcal{D}^{\prime}(M \times$ $M$ ) (see (2) in Theorem 21). This second analytic version (extended to $n$-dimensional spacetimes with $n \geq 2$ ) is the one usually nowadays adopted in perturbative aQFT, also including cosmological applications, starting from semiclassical versions of the Einstein equations (see [24] for a recent application). The Hadamard parametrix plays a special role in the definition of locally covariant Wick powers $[16,20]$ and in the study of quantum energy inequalities [10]. Kay-Wald's version of the Hadamard condition has been used by R. Verch to prove physically important properties of Hadamard states at algebraic level, like local quasi equivalence and local definiteness [38]. Using KayWald's definition, Sahlmann and Verch [34] extended the formalism to vector-valued quantum fields in a globally hyperbolic spacetime of dimension $n \geq 2$. There, also the equivalent microlocal formulation has been discussed and an extension of the theorem of propagation of Hadamard singularity has been established in the fashion of the original formulation [14] of that property of Hadamard states. We recommend [3] for a recent account on the wide spectrum of applications of Hadamard states (a pedagogical introduction to quasifree Hadamard states and their relevance in aQFT takes place in [20] therein).

The specific use of the Hadamard condition in the study of Hawking radiation can be traced back to [13], already before that the precise form of the Hadamard parametrix was stated in [19]. Though the microlocal version has been recently employed in applications to aQFT in black-hole background [9,35], the originary [19] version of the Hadamard condition has continued to play a crucial role to discuss the Hawking 
radiation [39], also in terms of a tunnelling process [6,29] (actually, those works only concern a local version of the Hadamard condition). See in particular the recent interesting work [22] on the Hawking radiation (and partially on the black-hole entropy) for a collapsing black-hole spherically symmetric spacetime, where the global Hadamard condition has been used.

An interesting global definition of Hadamard state has been recently discussed in [4] in terms of pseudo-differential operators and a different, but related, notion of global parametrix for globally hyperbolic spacetimes with compact Cauchy surfaces.

\subsection{A gap in the definition of $G_{\epsilon}^{T, n}$ and a proposal of solution}

The parametrix $G_{\epsilon}^{T, n}$ is evidently well defined on $\mathscr{Z}_{M}$, but there is no guarantee that it is also well defined on some open neighbourhood $\mathscr{O} \supset \mathscr{Z}_{M}$. Indeed, the open set $\mathscr{O}$ must also contain pairs $(p, q)$ which are not causally related and each such pair may be connected by many geodesic segments because Remark 13 does not apply. At this juncture, there is no explicit prescription to smoothly choose a unique geodesic segment for every such pair $(p, q)$ in order to have a well defined $\sigma(p, q)$, which, e.g. satisfies $\sigma(x, y)>0$ when $x \neq y$ are not causally related. The problem also arises in the definition of $\Delta(p, q)$ and $v^{n}(p, q)$ as they are computed using a geodesic segment joining $p$ and $q$ as said above.

Instead of attacking the problem directly by trying to establish the existence of a neighbourhood $\mathscr{O} \supset \mathscr{Z}_{M}$ where $\sigma$ and $G_{\epsilon}^{T, n}$ are well defined, we adopt a different strategy to circumvent the gap by employing the achievements of Sect. 2.2. The strategy relies on minimal modifications of original Kay-Wald's machinery. For this reason, in author's view, all important results established over the years that rely on Definition 15 (some of them quoted above) are correct.

Given a four-dimensional globally hyperbolic spacetime $(M, g)$ with a time orientation, choose a strong convex covering $\mathcal{C}$ of $M$, define the triple $(\mathscr{A}, \Gamma, \sigma)$ subordinated to $\mathcal{C}$ as in Theorem 10 and the set

$$
\mathscr{Z}_{M}^{\mathcal{C}}:=\{(x, y) \in M \times M \mid x, y \text { causally related, } J(x, y) \subset U \in \mathcal{C}\} .
$$

Notice that $\mathscr{A}$ is an open neighbourhood of $\mathscr{Z}_{M}^{\mathcal{C}}$ by construction.

(H1)' Define a (global) Hadamard parametrix subordinated to $\mathcal{C}$, for every natural $n$ and $\epsilon>0$, as

$$
\begin{aligned}
& G_{\epsilon}^{T, n, \mathcal{C}}(x, y):=\frac{1}{(2 \pi)^{2}} \\
& \quad\left[\frac{\Delta(x, y)^{1 / 2}}{\sigma(x, y)+2 i \epsilon t(x, y)+\epsilon^{2}}+v^{n}(x, y) \ln \left(\sigma(x, y)+2 i \epsilon t(x, y)+\epsilon^{2}\right)\right], \\
& \quad(x, y) \in \mathscr{A} .
\end{aligned}
$$

Above, $t(x, y):=T(x)-T(y)$, where $T: M \rightarrow \mathbb{R}$ is global smooth time function increasing towards the future, the branch cut of the logarithm is taken along 
the negative real axis, and the functions, $\sigma, \Delta$ and $v_{n}$ are the ones constructed out of $(\mathscr{A}, \Gamma, \sigma)$ starting from $\mathcal{C}$.

(H2) ${ }^{\prime}$ Given a smooth spacelike Cauchy surface $\Sigma$ of $(M, g)$ (with dimension $\geq 2$ ), a normal neighbourhood $N_{\mathcal{C}}$ of $\Sigma$ subordinated to $\mathcal{C}$ is an open set including $\Sigma$ and such that

(a) $\left(N_{\mathcal{C}},\left.g\right|_{N_{\mathcal{C}}}\right)$ is a globally hyperbolic spacetime and $\Sigma$ is a Cauchy surface of it;

(b) $(x, y) \in N_{\mathcal{C}} \times N_{\mathcal{C}}$ are causally related in $(M, g)$ iff $(x, y) \in \mathscr{Z}_{M}^{\mathcal{C}}$.

Lemma 17 Given a strong convex covering of $M$, every smooth spacelike Cauchy surface of $(M, g)$ admits a normal neighbourhood subordinated to $\mathcal{C}$.

Proof Use the same proof as the one of Lemma 2.2 of [19] with the only difference that all the used normal convex neighbourhoods must be taken in $\mathcal{C}$.

(H3)' Consider an open set $\mathscr{A}^{\prime} \subset N_{\mathcal{C}} \times N_{\mathcal{C}}$ which includes $\mathscr{Z}_{M}^{\mathcal{C}} \cap\left(N_{\mathcal{C}} \times N_{\mathcal{C}}\right)$ (i.e. the set of causally related pairs $\left.(x, y) \in N_{\mathcal{C}} \times N_{\mathcal{C}}\right)$ and such that its closure in $N_{\mathcal{C}} \times N_{\mathcal{C}}$ satisfies $\overline{\mathscr{A}}^{N_{\mathcal{C}} \times N_{\mathcal{C}}} \subset \mathscr{A} \cap\left(N_{\mathcal{C}} \times N_{\mathcal{C}}\right)$.

Remark $18 \mathscr{A}^{\prime}$ does exist because $\mathscr{Z}_{M}^{\mathcal{C}} \cap\left(N_{\mathcal{C}} \times N_{\mathcal{C}}\right)$ is closed in $N_{\mathcal{C}} \times N_{\mathcal{C}}$ (with the relative topology) and it is included in the open set $\mathscr{A} \cap\left(N_{\mathcal{C}} \times N_{\mathcal{C}}\right)$. (The set $\mathscr{Z}_{M}^{\mathcal{C}} \cap\left(N_{\mathcal{C}} \times N_{\mathcal{C}}\right)$ of causally related points in $N_{\mathcal{C}}$ is closed in $N_{\mathcal{C}} \times N_{\mathcal{C}}$ because $N_{\mathcal{C}}$ is globally hyperbolic and Lemma 22 in Chapter 14 of [31] is valid ${ }^{8}$.)

Finally, taking advantage of the smooth Urysohn lemma, choose a smooth function $\chi: N_{\mathcal{C}} \times N_{\mathcal{C}} \rightarrow[0,1]$ such that $\chi(x, y)=1$ for $(x, y) \in \overline{\mathscr{A}}^{N_{\mathcal{C}} \times N_{\mathcal{C}}}$ and $\chi(x, y)=0$ for $(x, y) \notin \mathscr{A} \cap\left(N_{\mathcal{C}} \times N_{\mathcal{C}}\right)$.

(H4)' With $\mathcal{C}, N_{\mathcal{C}}, T, \chi$ as above, we can give the definition of Hadamard state.

Definition 19 An algebraic state $\omega$ on the (Weyl $C^{*}$ or *) algebra of a real scalar KleinGordon field on $(M, g)$ is said to be globally Hadamard if the associated two-point function $\Lambda_{\omega}: C_{0}^{\infty}(M) \times C_{0}^{\infty}(M) \rightarrow \mathbb{C}$, satisfies the following requirement

$$
\begin{aligned}
& \Lambda_{\omega}\left(F_{1}, F_{2}\right)=\lim _{\epsilon \rightarrow 0^{+}} \int_{N_{\mathcal{C}} \times N_{\mathcal{C}}} \Lambda_{\epsilon}^{T, n, \mathcal{C}}(x, y) F_{1}(x) F_{2}(y) d \mu_{g}(x) d \mu_{g}(y), \\
& \forall F_{1}, F_{2} \in C_{0}^{\infty}\left(N_{\mathcal{C}}\right)
\end{aligned}
$$

where $\mu_{g}$ is the natural measure induced by $g$ on $M$ and

$$
\Lambda_{\epsilon}^{T, n, \mathcal{C}}(x, y)=\chi(x, y) G_{\epsilon}^{T, n, \mathcal{C}}(x, y)+H^{n}(x, y),
$$

for every natural $n$ and some associated functions $H^{n} \in C^{n}\left(N_{\mathcal{C}} \times N_{\mathcal{C}}\right)$.

\footnotetext{
8 The proof appearing in Lemma 3.3 in [32] of this fact seems to be wrong or incomplete, because $\sigma$ is not necessarily defined in the target points of the considered sequences, though it grasps the correct insight, with an appropriate use of the time separation function $\tau$ in place of $\sigma$ as in Chapter 14 of [31].
} 
Remark 20 An identity is of utmost physical interest: two parametrices subordinated to different strong convex coverings are however identical and also coincide with $G_{\epsilon}^{T, n}(x, y)$ in (8)

$$
G_{\epsilon}^{T, n, \mathcal{C}}(x, y)=G_{\epsilon}^{T, n, \mathcal{C}^{\prime}}(x, y)=G_{\epsilon}^{T, n}(x, y)
$$

when evaluated on causally related points $(x, y) \in N_{\mathcal{C}} \cap N_{\mathcal{C}^{\prime}}$. In fact, in the said hypothesis, it simultaneously holds $(x, y) \in J(x, y) \subset C \in \mathcal{C}$ and $(x, y) \in J(x, y) \subset$ $C^{\prime} \in \mathcal{C}^{\prime}$ and thus, according to Remark 13, the geodesic segments joining $x$ and $y$ in $C$ and $C^{\prime}$, respectively, coincide. Finally the parametrices coincide as well in view of Remark 14. What happens to $G_{\epsilon}^{T, n, \mathcal{C}}(x, y)$ for non-causally related points is physically irrelevant and it permits an arbitrary choice of the function $\chi$ appearing in $\chi(x, y) G_{\epsilon}^{T, n, \mathcal{C}}(x, y)$. A change of the function $\chi$ can be reabsorbed in a change of the functions $H^{n}$. That is a consequence of the fact that, for $x \neq y$ non-causally related, $\sigma(x, y)>0$ and no singularity (for $\epsilon \rightarrow 0^{+}$) shows up in the parametrix $G_{\epsilon}^{T, n, \mathcal{C}}(x, y)$. In other words, the parametrix viewed as a distribution is actually a smooth function for non-causally related arguments. All that was discussed and clearly emphasized in [19] referring to the parametrix $G_{\epsilon}^{T, n}$. Unfortunately these properties of $G_{\epsilon}^{T, n}$ rely also on a good behaviour of $\sigma$ in the whole open neighbourhood $\mathscr{O}$ (and $\mathscr{O}^{\prime}$ ) which is not proved to exist.

\subsection{Independence of the choices of $\mathcal{C}, N_{\mathcal{C}}, T, \chi$ and nice interplay with the microlocal formulation}

What remains to be demonstrated is that the given definition of Hadamard state does not depend on the choice of $\mathcal{C}, N_{\mathcal{C}}, T, \chi$ and it corresponds to the microlocal formulation [32]. Radzikowski [32] aimed to establish that a state of a real Klein-Gordon field in a globally hyperbolic spacetime $(M, g)$ is Hadamard in the sense of [19] if and only if it satisfies the microlocal spectral condition (14). (Actually, it was done when also assuming the fair hypothesis that the two-point function $\Lambda$ is a distribution of $\mathcal{D}^{\prime}(M \times M)$.) As a matter of fact, this result gave rise to an alternative definition of Hadamard state.

The presentation of the Hadamard condition in the original sense of [19] in [32,33] is affected by the issue pointed out above (in the proof of Lemma 3.1 in [32] in particular) since [32] includes a faithful summary of relevant ideas and notions appearing in [19].

We argue that the statement of Theorem 5.1 in [32] which establishes the equivalence of the two formulations is however valid when assuming our definition of Hadamard state according to $(\mathrm{H} 1)^{\prime}-(\mathrm{H} 4)^{\prime}$. Let us re-state here part of Radzikowski's equivalence theorem (excerpt from Theorem 5.1 [32] with notations adapted to our paper).

Theorem 21 Let $(M, g)$ be a smooth, time oriented, four-dimensional globally hyperbolic spacetime and $\Lambda \in \mathcal{D}^{\prime}(M \times M)$, define the Klein-Gordon operator $P:=$ $-\square+m^{2}: C^{\infty}(M) \rightarrow C^{\infty}(M)$ for some real valued $m^{2} \in C^{\infty}(M)$. Choose $\mathcal{C}, T, \chi, N_{\mathcal{C}}$ as above. Then, the following conditions are equivalent.

(1) $\Lambda$ satisfies what follows. 
(a) The global Hadamard condition in Definition 19 (referring to the given choice of $\mathcal{C}, T, \chi, N_{\mathcal{C}}$ ),

(b) its antisymmetric part is $\frac{i}{2}\left(\Delta_{A}-\Delta_{R}\right)$ (where $\Delta_{A / R}: C_{0}^{\infty}(M) \rightarrow C^{\infty}(M)$ are the advanced/retarded Green operators of $P$ ),

(c) $\Lambda\left(P F \otimes F^{\prime}\right)=\Lambda\left(F \otimes P F^{\prime}\right)=0$ for all real-valued $F, F^{\prime} \in C_{0}^{\infty}(M)$.

(2) $\Lambda$ satisfies what follows.

(a)' The microlocal spectral condition

$$
\begin{aligned}
& W F(\Lambda)= \\
& \left\{\left(\left(x_{1}, k_{1}\right),\left(x_{2}, k_{2}\right) \in T^{*} M \backslash \mathbf{0} \times T^{*} M \backslash \mathbf{0} \mid\left(x_{1}, k_{1}\right),\left(x_{2},-k_{2}\right), k_{1} \triangleright 0\right\},\right.
\end{aligned}
$$

(b) its antisymmetric part is $\frac{i}{2}\left(\Delta_{A}-\Delta_{R}\right)$,

(c) $\Lambda\left(P F \otimes F^{\prime}\right)=\Lambda\left(F \otimes P F^{\prime}\right)=0$ for all real-valued $F, F^{\prime} \in C_{0}^{\infty}(M)$.

((b) and (c) are in particular valid if $\Lambda=\Lambda_{\omega}$ for an algebraic state $\omega$ on the (Weyl $C^{*}$ or $^{*}$ ) algebra of a real Klein Gordon quantum field.)

Equivalence of (1) and (2) is still valid with the following changes. (b) and (c) in (1) are true $\bmod C^{\infty},(b)$ in (2) is true $\bmod C^{\infty}$, and (c) is omitted from (2).

Sketch of Proof. The proof of Theorem 5.1 [32] uses both microlocal analysis arguments and some results from [19]. Concerning definitions and facts established in [19], it is assumed that (i) the parametrix $G_{\epsilon}^{T, n}(x, y)$ has the known structure in terms of $\sigma, \Delta, v^{n}$ in a covering of normal convex neighbourhoods as stated in [19], (ii) the Hadamard expansion is well-behaved on the open neighbourhood $\mathscr{O}^{\prime}$ of the causally related points in $N \times N$, where $\sigma(x, y)>0$ for $x \neq y$ which are not causally related (more precisely it takes the standard form $\sigma\left(x, x^{\prime}\right)=-\left(y^{0}\left(x^{\prime}\right)\right)^{2}+\sum_{\alpha=1}^{3}\left(y^{\alpha}\left(x^{\prime}\right)\right)^{2}$ in Riemannian normal coordinates $y^{0}, y^{1}, y^{2}, y^{3}$ centred at $x$ ), and (iii) the definition of Hadamard state according to [19] is independent from the choice of the Cauchy temporal function $T$. This proof of independence appears in Appendix B of [19] and it can be recast without changes for our definition of Hadamard state based on the parametrix $G_{\epsilon}^{T, n, \mathcal{C}}(x, y)$ and a normal neighbourhood $N_{\mathcal{C}}$. In summary, replacing $\mathscr{O}^{\prime}$ for $\mathscr{A}^{\prime}$, using the fact that $G_{\epsilon}^{T, n, \mathcal{C}}(x, y)$ has the same local structure as $G_{\epsilon}^{T, n}(x, y)$ in terms of $\sigma$ and the Hadamard expansion coefficients and is well-behaved on $\mathscr{A}$, and exploiting independence of the definition from the choice of $T$, the proof of Theorem $5.1[32]$ is valid as it stands for Definition 19 of Hadamard state ${ }^{9}$.

Corollary 22 The definition of Hadamard state in Definition 19 (based on (H1)'-(H4)' and assuming $(b)$ and $(c)$ of Theorem 21 for $\Lambda_{\omega}$ ) does not depend on the choices of $\mathcal{C}, N_{\mathcal{C}}, T, \chi$ if $\Lambda_{\omega} \in \mathcal{D}^{\prime}(M \times M)$.

\footnotetext{
9 As is known, the proof of Theorem 5.1 in [32] has a gap. It is the content of the three lines immediately before the proof of (ii) $3 \Rightarrow 2$ on p. 547. This gap was closed in several independent works, in particular (but not only) [34] and [20]. In the latter, only the microlocal analysis approach was exploited and thus without relation with the issue with [19] definition of Hadamard state. See Remark 23 in [20] for a summary on this subject.
} 
Proof Item (2) above does not depend on the choices of $\mathcal{C}, N_{\mathcal{C}}, \chi$. (Independence from the choice of $T$ was independently established in the proof of Theorem 21 using the same proof as in [19].)

To conclude we observe that, following [33], an algebraic state $\omega$ on a KleinGordon quantum field on a spacetime $(M, g)$ is said to be locally Hadamard if there is a (normal convex) neighbourhood $U$ of every point where, for every natural $n$, the two-point function of the state $\Lambda_{\omega}$ can be decomposed as in (10) (i.e. (13)) with $\Lambda_{\omega}$ in place of $\Lambda^{T, n}$, for $\chi=1$ and $H^{n} \in C^{n}(U \times U)$. It is possible to prove that a state $\omega$ such that $\Lambda_{\omega} \in \mathcal{D}^{\prime}(M \times M)$ satisfies (b) in Theorem 21 is locally Hadamard in a four-dimensional globally hyperbolic spacetime if and only if it is globally Hadamard. It was established in Theorem 9.2 in [33] using only the microlocal definition (i.e. (2) in Theorem 21) of Hadamard state and thus that result is valid also with our definition of (global) Hadamard state.

Acknowledgements I am grateful to Franco Cardin, Claudio Dappiaggi, Nicolò Drago, Nicola Pinamonti, and Miguel Sànchez for various discussions over the years on subjects somehow related to the content of this work. I thank Chris Fewster and Miguel Sànchez for several technical discussions and suggestions about this paper. I am grateful to the two anonymous referees for a number of suggestions and remarks of various nature which really helped me improve the presentation of this work. I am finally grateful to C. Fewster, F. Kurpicz, N. Pinamonti, and R. Verch who, directly or indirectly, encouraged the author to write down this quite technical note.

Funding Open access funding provided by Universitá degli Studi di Trento within the CRUI-CARE Agreement.

Open Access This article is licensed under a Creative Commons Attribution 4.0 International License, which permits use, sharing, adaptation, distribution and reproduction in any medium or format, as long as you give appropriate credit to the original author(s) and the source, provide a link to the Creative Commons licence, and indicate if changes were made. The images or other third party material in this article are included in the article's Creative Commons licence, unless indicated otherwise in a credit line to the material. If material is not included in the article's Creative Commons licence and your intended use is not permitted by statutory regulation or exceeds the permitted use, you will need to obtain permission directly from the copyright holder. To view a copy of this licence, visit http://creativecommons.org/licenses/by/4.0/.

\section{References}

1. Brunetti, R., Fredenhagen, K.: Microlocal analysis and interacting quantum field theories: renormalization on physical backgrounds. Commun. Math. Phys. 208, 623-661 (2000)

2. Brunetti, R., Fredenhagen, K., Köhler, M.: The microlocal spectrum condition and Wick polynomials of free fields on curved space-times. Commun. Math. Phys. 180, 633-652 (1996)

3. Brunetti, R., Dappiaggi, C., Fredenhagen, K., Yngvason, J. (eds.): Advances in Algebraic Quantum Field Theory. Springer, New York (2015)

4. Capoferri, M., Dappiaggi, C., Drago, N.: Global wave parametrices on globally hyperbolic spacetimes. J. Math. Anal. Appl. 490(2), 124316 (2020)

5. Cardin, F., Marigonda, A.: Global world functions. J. Geom. Symmetry Phys. 2, 1-17 (2004)

6. Collini, G., Moretti, V., Pinamonti, N.: Tunnelling black-hole radiation with $\phi^{3}$ self-interaction: oneloop computation for Rindler Killing horizons Lett. Math. Phys. 104, 217-232 (2014)

7. Dappiaggi, C., Fredenhagen, K., Pinamonti, N.: Stable cosmological models driven by a free quantum scalar field. Phys. Rev. D 77, 104015 (2008)

8. Dappiaggi, C., Moretti, V., Pinamonti, N.: Distinguished quantum states in a class of cosmological spacetimes and their Hadamard property. J. Math. Phys. 50, 062304 (2009) 
9. Dappiaggi, C., Moretti, V., Pinamonti, N.: Rigorous construction and Hadamard property of the Unruh state in Schwarzschild spacetime. Adv. Theor. Math. Phys. 15, 355-447 (2011)

10. Fewster, C.J., Smith, C.J.: Absolute quantum energy inequalities in curved spacetime. Ann. Henri Poincaré 9, 425-455 (2008)

11. Fewster, C.J., Verch, R.: Stability of quantum systems at three scales: passivity, quantum weak energy inequalities and the microlocal spectrum condition. Commun. Math. Phys. 240, 329-375 (2003)

12. Fewster, C.J., Verch, R.: The necessity of the Hadamard condition. Class. Quantum Gravity 30, 235027 (2013)

13. Fredenhagen, K., Haag, R.: On the derivation of Hawking radiation associated with the formation of a black hole. Commun. Math. Phys. 127, 273 (1990)

14. Fulling, S.A., Sweeny, M., Wald, R.M.: Singularity structure of the two-point function in quantum field theory in curved spacetime. Commun. Math. Phys. 63, 257-264 (1978)

15. Hack, T.P., Moretti, V.: On the stress-energy tensor of quantum fields in curved spacetimes-comparison of different regularization schemes and symmetry of the Hadamard/Seeley-DeWitt coefficients. J Phys A Math Theor 45(37), 374019 (2012)

16. Hollands, S., Wald, R.M.: Local Wick polynomials and time ordered products of quantum fields in curved spacetime. Commun. Math. Phys. 223, 289-326 (2001). arXiv:gr-qc/0103074

17. Hollands, S., Wald, R.M.: Existence of local covariant time ordered products of quantum fields in curved spacetime. Commun. Math. Phys. 231, 309-345 (2002)

18. Kay, B.S.: A uniqueness result for quasi-free KMS states. Helv. Phys. Acta 58, 1017-1029 (1985)

19. Kay, B.S., Wald, R.M.: Theorems on the uniqueness and thermal properties of stationary, nonsingular, quasifree states on spacetimes with a bifurcate Killing horizon. Phys. Rep. 207(2), 49-136 (1991)

20. Khavkine, I., Moretti, V.: Algebraic QFT in curved spacetime and quasifree Hadamard states: an introduction, Chapter 5 of [BDFY15]

21. Kobayashi, K., Nomizu, S.: Foundations of Differential. Geometry, vol. I. (1996)

22. Kurpicz, F., Pinamonti, N., Verch, R.: Temperature and entropy-area relation of quantum matter near spherically symmetric outer trapping horizons. Lett. Math. Phys. (2021) in print. arXiv preprint arXiv:2102.11547

23. Lee, J.: Introduction to Riemannian Manifolds. Springer, New York (2018)

24. Meda, P., Pinamonti, N., Siemssen, D.: Existence and uniqueness of solutions of the semiclassical Einstein equation in cosmological models. In: Annales Henri Poincaré, pp. 1-51. Springer, New York (2021)

25. Michael, E.: Yet another note on paracompact spaces. Proc. Am. Math. Soc. 10(2), 309-314 (1959)

26. Minguzzi, E.: Lorentzian causality theory. Living reviews in relativity 22(1), 1-202 (2019)

27. Moretti, V.: Comments on the stress-energy tensor operator in curved spacetime. Commun. Math. Phys. 232, 189-221 (2003)

28. Moretti, V.: Quantum out-states holographically induced by asymptotic flatness: invariance under spacetime symmetries, energy positivity and Hadamard property. Commun. Math. Phys. 279, 31-75 (2008)

29. Moretti, V., Pinamonti, N.: State independence for tunneling processes through black hole horizons. Commun. Math. Phys. 309, 295-311 (2012)

30. Nomizu, K., Ozeki, H.: The existence of complete Riemannian metrics. Proc. Am. Math. Soc. 12(6), 889-891 (1961)

31. O`Neill, B.: Semi-Riemannian Geometry With Applications to Relativity. Academic Press, Cambridge (1983)

32. Radzikowski, M.J.: Microlocal approach to the Hadamard condition in quantum field theory on curved space-time. Commun. Math. Phys. 179, 529-553 (1996)

33. Radzikowski, M.J., Verch, R.: A local-to-global singularity theorem for quantum field theory on curved space-time. Commun. Math. Phys. 180, 1-22 (1996)

34. Sahlmann, H., Verch, R.: Microlocal spectrum condition and Hadamard form for vector-valued quantum fields in curved space-time. Rev. Math. Phys. 13, 1203-1246 (2001)

35. Sanders, K.: On the construction of Hartle-Hawking-Israel states across a static bifurcate Killing horizon. Lett. Math. Phys. 105, 575-640 (2015)

36. Shubin, M.A.: Spectral theory of elliptic operators on non-compact manifolds, dans Méthodes semiclassiques. Astérisque 1(207), 74 (1992)

37. Stone, A.H.: Paracompactness and product spaces. Bull. Am. Math. Soc. 54, 977-982 (1948) 
38. Verch, R.: Local definiteness, primarity and quasiequivalence of quasifree Hadamard quantum states in curved spacetime. Commun. Math. Phys. 160, 507-536 (1994)

39. Wald, R.M.: Quantum Field Theory in Curved Spacetime and Black Hole Thermodynamics. Chicago Lectures in Physics, University of Chicago Press, Chicago (1994)

Publisher's Note Springer Nature remains neutral with regard to jurisdictional claims in published maps and institutional affiliations. 